\title{
Platinum-Resistant Fallopian Tube
}

\section{Carcinoma}

National Cancer Institute

\section{Source}

National Cancer Institute. Platinum-Resistant Fallopian Tube Carcinoma. NCI Thesaurus.

Code C157621.

Fallopian tube carcinoma that is resistant to platinum therapy. 